\title{
On the Use of Orthogonal Transformations in the Reduction of Paleomagnetic Data
}

\author{
V.A. SCHMIDT \\ Department of Earth and Planetary Sciences, University \\ of Pittsburgh, Pittsburgh, Pa. 15260, U.S.A.
}

(Received August 8, 1974)

\begin{abstract}
A modified Eulerian orthogonal transformation is extremely useful in transforming the magnetization vector of a rock sample from the sample coordinate system as measured in a magnetometer to the ancient coordinate system at the sampling site, especially if complicated folding has occurred since the rock was magnetized. The method is adaptable to a wide variety of sample collecting and marking conventions. The form and function of the transformation, which has been widely used in other fields, is reviewed and is applied to the following situations of interest in paleomagnetism: transformation from sample to site coordinates; transformation of sample coordinates if the reference or marking plane is oblique to the sample coordinate system; and removal of the effects of horizontal or plunging folds. An earlier treatment of this latter problem by Zotkevich (1972) is shown to be incorrect. Additional applications of the transformation are made to the display of paleomagnetic data. The transformation has many potential applications in structural geology.
\end{abstract}

\section{Introduction}

The goal of most paleomagnetic experiments is the reconstruction of the ancient Earth's magnetic field vector at a given sampling site. Seldom, however, is the paleomagnetic vector of the rock formation determined in situ. Instead, an arbitrary reference plane fixed to the sample is oriented with respect to a coordinate system (usually north, east, and straight down) which we shall refer to as the site coordinates. The actual measurement of the magnetic vector is generally done in the laboratory with respect to a coordinate system defined by the sample's reference plane. We shall refer to this coordinate system as the sample coordinates.

The transformation of the magnetic vector from sample to site coordinates by any one of several methods is not difficult, but sometimes the situation is complicated by intervening geological disturbances or sampling difficulties that make it necessary to transform to intermediate coordinate systems in order to obtain the true orientation of the ancient magnetic vector (which we shall refer to as the ancient coordinates). When such complications are present, paleomagnetists generally have turned to two methods. The first uses a stereographic net 
and allows the successive rotations of coordinates (or alternatively, of the magnetic vector itself) to be made once the desired rotations have been established. The second requires the solution of the problem via spherical trigonometry.

Methods based on the use of stereographic nets have the distinct advantages of simplicity and flexibility of application. Unfortunately, being graphical constructions, they have the disadvantages of introducing graphical inaccuracies and of being tedious and time-consuming.

Any problem consisting of a rotation of coordinates may be solved by the proper application of spherical trigonometry, but some situations of interest in paleomagnetism are of such complexity that solutions are extremely difficult to obtain and the likelihood of error is high.

What is needed is a method that combines the simplicity and flexibility of the graphical methods with the requirements of computability. Fortunately, such a method exists in the form of an orthogonal transformation which utilizes rotations about Eulerian angles. In wide use in mathematics and classical mechanics, this simple transformation executes a rotation of coordinates about any arbitrary axis and thus performs the same functions as the graphical methods cited above. The use of this transformation for paleomagnetic (or other geological) problems enjoys the following advantages:

(a) Once the desired rotations are defined, the problem is solved by a routine application of the transformation.

(b) Complicated problems involving successive rotations about different axes (such as the rectification of a plunging fold), may be solved by successive routine applications of the same transformation.

(c) The transformation operates solely within Cartesian coordinates. As a result, all the complications of spherical trigonometry are avoided. This is particularly convenient since raw magnetometer data is already Cartesian. Conversion to spherical coordinates is made only as a final step, in order to present results in terms of familiar quantities such as inclination and declination.

(d) The most general form of the transformation may be readily and simply constructed as a computer subroutine.

(e) The accuracy of transformation can be very high. Using seven- or eight-digit precision arithmetic, several successive transformations can be carried out with accuracies of better than a tenth of a degree.

\section{Definition of the Transformation}

A coordinate system $(x, y, z)$ may be transformed into any other $\left(x^{\prime \prime \prime}, y^{\prime \prime \prime}\right.$, $\left.z^{\prime \prime \prime}\right)$ by three successive rotations: of angle $\phi$ about the $z$ axis, of angle $\theta$ about the $y^{\prime}$ axis, and of angle $\phi$ about the $z^{\prime \prime}$ axis* as shown in Fig. 1. These three

* Actually, the Euler angle $\theta$ is usually defined as a rotation about $x^{\prime}$. As a matter of convenience, this has been modified to a rotation about $y^{\prime}$ since rotations about the strike are common 

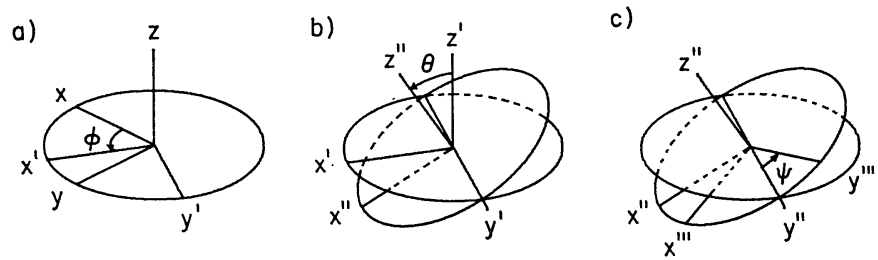

Fig. 1. The modified Euler angles $\phi, \theta, \dot{\psi}$ are shown as positive rotations of the coordinate axes. The magnetization vector, which is not shown, remains fixed in orientation throughout the process.

independent parameters are all that are necessary to specify the most general rotation of coordinates (Euler's Theorem). If the coordinates of a vector in the original coordinate system are $x, y, z$ then the orthogonal transformation is defined by:

$$
\begin{aligned}
x^{\prime \prime \prime}= & (\cos \phi \cos \theta \cos \phi-\sin \phi \sin \phi) x \\
& +(\cos \phi \cos \theta \sin \phi+\sin \phi \cos \phi) y-(\cos \phi \sin \theta) z \\
y^{\prime \prime \prime}= & -(\sin \phi \cos \theta \cos \phi+\cos \phi \sin \phi) x \\
& -(\sin \phi \cos \theta \sin \phi-\cos \phi \cos \phi) y+(\sin \phi \sin \theta) z \\
z^{\prime \prime \prime}= & (\sin \theta \cos \phi) x+(\sin \theta \sin \phi) y+(\cos \theta) z .
\end{aligned}
$$

The reader is referred to the standard texts** for the mode of derivation. The transformation is valid for all possible values of $\phi, \theta$, and $\phi$.

The operation of the orthogonal transformation may be visualized in either of two equivalent ways. Each has its own particular usefulness and we will use one or the other in the various examples given below, depending solely on which provides the easiest visualization. The rotation conventions must be clearly understood, however, since they are different for the two approaches.

Fixed vector; rotation of coordinate system: This is the case shown in Fig. 1. The vector to be transformed (not shown) is fixed in space while the coordinate system is rotated. Each of the three rotation angles $\phi, \theta, \phi$ are considered to be positive when its corresponding rotation of axes is counter-clockwise when viewed from the positive end of the rotation axis. The three particular rotations shown in Fig. 1 are all positive. Negative as well as positive rotations of any magnitude are allowed for each of the three angles.

Fixed coordinate system; rotation of vector: In some cases it may be preferable to rotate the vector itself, retaining the original coordinate system fixed in space. The three successive rotations are once again defined as of angle $\phi$ about the $z$ axis, followed by $\theta$ about the $y^{\prime}$ axis, which is followed by $\phi$ about the $z^{\prime \prime}$ axis.

and many paleomagnetism laboratories have assigned the $y$ axis to the strike. Equation (1) reflects this modification.

** Discussed in many introductory texts. A particularly good discussion is given by Goldstein (1950). 
Now, however, each of the three rotation angles are considered to be positive when its corresponding rotation of the vector is clockwise when viewed from the positive end of the rotation axis. The equivalence of the two visualizations is obvious: it all depends on whether the observer chooses to stay fixed with the vector or to ride with the coordinate system during the rotation operations.

\section{Applications}

In each application, the following conventions are used:

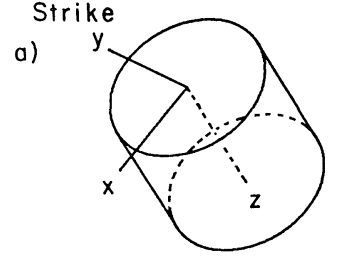

b)

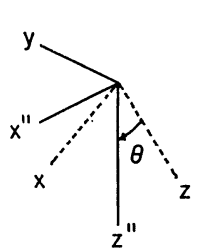

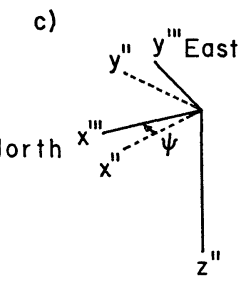

Fig. 2. (a) The sample is shown in its field orientation. The $y$ axis of the sample coordinate system is assumed to be the strike of the reference plane, which is the $x y$ plane. (b), (c) Only two rotations are needed to transform to site coordinates.

Sample coordinates (See Fig. 2):

$x$ : the down-dip direction of the reference plane

$y$ : the strike direction of the reference plane (looking up-dip, the strike always points to the left)

$z$ : straight down into the sample from the reference plane.

Site or ancient coordinates:

$x$ : north

$y$ : east

$z$ : straight down.

Other conventions for sample marking are easily accommodated as discussed below. It is important, however, that all strikes are measured (or converted) according to the convention given above.

A. Conversion of sample to site coordinates: We shall keep the magnetization vector (and sample) fixed and rotate the coordinate system. Fig. 2a shows the sample (with its coordinate system $x, y, z$ ) in its sampling site orientation. Since the strike of the reference plane is already horizontal by definition, we may dispense with the first rotation about the $z$ axis and proceed directly to the rotation about the $y^{\prime}$ axis in order to bring the $x, y$ plane horizontal and the $z$ axis straight down (Fig. 2b). The angle of rotation $\theta$ is, again by definition, the dip angle and is positive. Since the angle between north and the $y^{\prime \prime}$ axis is the measured strike angle, a rotation of $\phi=\left(90^{\circ}-\right.$ strike $)$ will bring the $x$ axis into coincidence with the north direction and the $y$ axis into coincidence with the 
east direction (Fig. 2c). Thus the problem is solved with a single application of the transformation (1) with:

$$
\begin{aligned}
& \phi=0, \\
& \theta=\text { dip of reference plane, } \\
& \phi=90^{\circ} \text {-strike of reference plane. }
\end{aligned}
$$

It should be emphasized that this procedure is valid for any possible values for dip and strike, so long as the conventions defined above are used. The dip may even exceed $90^{\circ}$, such as in the case where a sample core is drilled upward into an overhang.

It should now be clear how other marking conventions are to be accommodated. For example, if the $x$ axis in the sample coordinate system is assigned to the up-dip direction on the reference plane (as some laboratories do), an initial rotation of $\phi=180^{\circ}$ about the $z$ axis instead of $\phi=0$ will convert between the two conventions. The rotations $\theta$ and $\phi$ remain unchanged. Regardless of the convention used, it is of course imperative to work solely in right-handed coordinate systems.

B. Rectification of a simple bedding plane tilt: The previous operation has yielded the coordinates of the magnetization vector in the present site coordinate system. If the sample itself has not been disturbed since the time of its magnetization, then the site coordinates are equivalent to the ancient coordinates and no further treatment is necessary. If, however, geological evidence indicates that the sample has been rotated about a horizontal axis since its magnetization, then we must undo this rotation, and transform the magnetization vector into the ancient coordinate system. The geological evidence is usually in the form of a simple folding of initially horizontal strata about a horizontal axis. A simple fold is defined as a ruled surface whose generating line remains everywhere parallel to the fold axis. Both this application and the next are restricted to simple folds. It is assumed that the strike and dip of the bedding plane are measured at the sampling site. The bedding plane and the reference plane need not be coincident.

The measured bedding strike should be nearly parallel to the local folding axis. Any discrepancy between these two directions will give a rough idea of the error introduced by the assumption of a simple horizontal fold.

The problem may be defined as follows: in order to restore the bedding plane to the horizontal, we must rotate the bedding plane about its strike by an angle equal to its dip. In order to accomplish this via the orthogonal transformation, we must first go to an intermediate coordinate system in which the strike lies along the $y$ axis. It is easier to visualize this process in terms of rotating the bedding plane (and the magnetization vector with it) and keeping the coordinate system fixed. Figure 3 shows the geometry of the situation. The first rotation of angle $\phi$ about the $z$ axis brings the bedding strike into coincidence 
a)

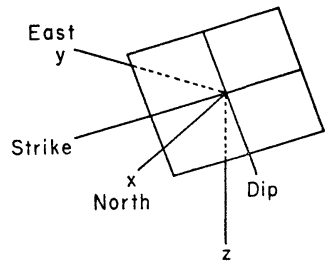

c)

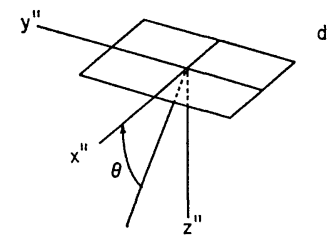

b)

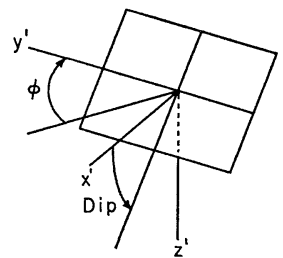

d)

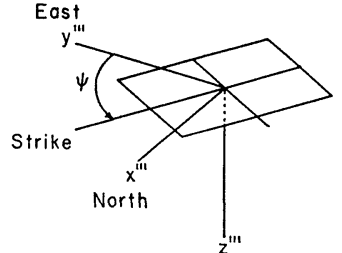

Fig. 3. In restoring a dipping bedding plane to its original attitude, we choose to keep the coordinate system fixed and to rotate the bedding plane instead. The magnetization vector is fixed in relation to the bedding plane.

with the $y^{\prime}$ axis. Since this is a counter-clockwise rotation of the vector as seen from the $+z$ axis, the rotation is in the negative sense, and so $\phi=$ bedding strike $-90^{\circ}$. The next rotation about $y^{\prime}$ (the strike) is $\theta=-$ bedding dip, since the rotation is again in the negative sense. The plane is now horizontal, and the final rotation about $z^{\prime \prime}$ of $\phi=-\dot{\phi}=90^{\circ}$ - bedding strike (a positive rotation) restores the strike to its original orientation. Hence, once the vector has been converted to site coordinates by the method given in application A above, the transformation to ancient coordinates (removal of the bedding plane tilt) is accomplished by an additional application of the transformation with:

$$
\begin{aligned}
& \phi=\text { bedding strike }-90^{\circ}, \\
& \theta=- \text { bedding dip, } \\
& \phi=90^{\circ} \text { - bedding strike. }
\end{aligned}
$$

ZotKevich (1966) has treated the present example using a combination of spherical trigonometry, graphical constructions, and at one point he even introduces a special case of the orthogonal transformation used here. Though cumbersome, his method appears to give correct results.

C. Rectification of a bedding plane tilt on the limb of a plunging fold: Once again, we shall rotate the vector (along with the strata) and keep the coordinate system fixed. This is considerably more complicated than the previous case, since two distinct transformations will be necessary. The first will remove the plunge from the fold, and the second will open out the fold to the original horizontal plane.

ZotKeVICH (1972) has attempted this problem also, this time using a purely spherical trigonometric approach. He arrives at the curious conclusion that failure to take account of the plunge to the fold (that is, attempting to solve the 


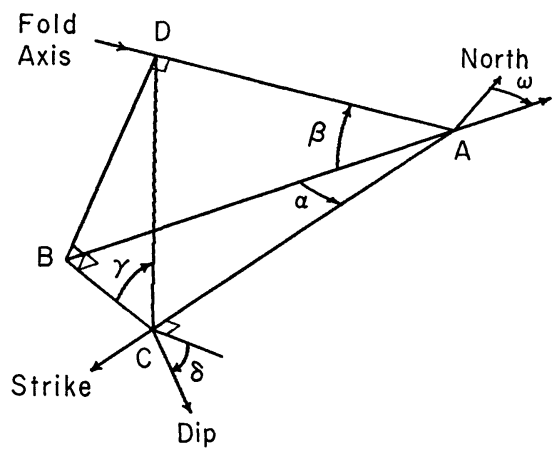

Fig. 4. The geometry of a plunging fold. The fold axis makes an angle $\beta$ with the horizontal plane ABC, and the strike and dip of the bedding plane are measured at the sampling site at $C$.

present example by the method of the preceding one) will introduce error into the inclination of the vector but not into the declination. It is not hard to see that this result is incorrect. Since the magnetization vector is fixed in relation to the bedding plane, and since the bedding plane is restored to the horizontal whether or not the angle of plunge is taken into account, then it should be clear that the resulting inclination of the vector will be the same, regardless of whether or not the plunge is taken into account. As a result, the error is introduced solely into the declination, and not at all into the inclination. The method which we shall now develop does, in fact, verify this conclusion.

Figure 4 gives the essential geometry of a plunging fold, which need not be symmetric. The fold axis AD plunges with angle $\beta$ to the horizontal plane $A B C$, while the sampling site is on a limb of the fold at $\mathrm{C}$. The line $\mathrm{AC}$ is the bedding strike as measured at the sampling site and makes an angle $\alpha$ with $\mathrm{AB}$, the horizontal projection of the fold axis, which in turn makes an angle $\omega$ with North. We begin with the magnetization vector expressed in site coordinates. The first step is to remove the plunge by rotating the entire construction in Fig. 4 about the horizontal line $\mathrm{BC}$, where the plane of the triangle $\mathrm{BCD}$ is perpendicular to the fold axis. Note that this will bring the triangle BCD into a vertical plane, but will take the measured line of bedding dip out of the vertical plane. In this new configuration, the bedding plane at $\mathrm{C}$ will have a new dip along $\mathrm{DC}$ with dip angle $\gamma$. Note that DC is not colinear with the original line of dip and so $\gamma$ is in general different from the measured dip $\delta$. From Fig. 4 we obtain

$$
\tan \gamma=\frac{\sin \beta}{\tan \alpha}
$$

from which we may determine $\gamma$. Zоткеvich (1972) derives the useful relation

$$
\tan \beta=\sin \alpha \tan \delta \text {. }
$$

Combining Eqs. (4) and (5) yields

$$
\tan \gamma=\tan \delta \cos \beta \cos \alpha,
$$


showing the relation between $\gamma$ and $\delta$ more clearly.

The final step involves rotating the bedding plane ACD through angle $\gamma$ about the fold axis in order to bring the bedding plane into a horizontal configuration. This last transformation is equivalent to that given in application $\mathbf{B}$, with the angle $\gamma$ substituted for the bedding dip. It is more efficient, however, to partially combine the two transformations.

Let us now define the appropriate rotations for removing a plunging fold. The first is a positive rotation of the magnetization vector (and the strata with it) about the $z$ axis with $\phi_{1}=\omega$ to bring the fold axis along a north-south line ( $x^{\prime}$ axis) and to bring the line $\mathrm{BC}$ along the $y^{\prime}$ axis. The next rotation about $y^{\prime}$ removes the plunge and is a negative rotation, hence $\theta_{1}=-\beta$. Now, instead of rotating about $z^{\prime \prime}$ by $-\omega$ to return the now-horizontal fold axis to its original declination, let us rotate by $\phi_{1}=90^{\circ}$, which leaves the fold axis pointing along the $-y$ axis. The fold is now in position for the next transformation, that of restoring the bedding plane to the horizontal. As a result, we may dispense with the first rotation about the new $z$ axis by setting $\phi_{2}=0$. Next the beds are rotated through the angle $\gamma$ to a horizontal attitude. If the sampling site is on the right limb (looking down the plunging axis) of an anticline or on the left limb of a syncline, the rotation is in the negative sense and $\theta_{2}=-\gamma$. If it is on the left limb of an anticline or the right limb of a syncline, the rotation is positive and $\theta_{2}=+\gamma$. The bedding plane is now horizontal and the final rotation about $z^{\prime \prime}$ of $\psi_{2}=-90^{\circ}-\omega$ restores the fold axis to its original declination.

To summarize the entire process:

1) The following measurements should be made in the field:

$\omega$ : declination of fold axis, where the fold axis points in the down-plunging direction $\left(0 \leq \omega \leq 360^{\circ}\right)$

$\beta$ : angle of plunge of fold axis $\left(0 \leq \beta \leq 90^{\circ}\right)$

$\alpha$ : the angle between the bedding strike at the sampling site and the horizontal projection of the fold axis $\left(0 \leq \alpha \leq 90^{\circ}\right)$

$\delta$ : the bedding dip at the sampling site $(0 \leq \delta)$.

2) The angle $\gamma$ is determined from Eq. (4).

3) Starting with the magnetization vector expressed in terms of site coordinates (as obtained from application A), the first transformation is applied with:

$$
\begin{aligned}
& \phi_{1}=\omega, \\
& \theta_{1}=-\beta, \\
& \phi_{1}=+90^{\circ} .
\end{aligned}
$$

4) The transformed coordinates are subjected to a second transformation with: $\phi_{2}=0$, $\theta_{2}= \begin{cases}-\gamma & \text { for right limb of anticline or left limb of syncline, } \\ +\gamma & \text { for left limb of anticline or right limb of syncline, }\end{cases}$ $\phi_{2}=-90^{\circ}-\omega$. 
The magnetization vector is now in its correct ancient orientation.

The case of an overturned limb is correctly handled by the above procedure so long as the convention is used that the bedding dip of an overturned limb will always be greater than $90^{\circ}$. Obviously, the original sequence of the beds in an overturned fold must also be known in order to satisfy the requirements for determining $\theta_{2}$ in Eq. (8).

As in the previous example, the above procedure is strictly valid only for a simple fold. Fortunately, Eq. (5) allows us to check this assumption if $\beta, \alpha$, and $\delta$ have all been measured in the field. The discrepancy in $\alpha$ as calculated from Eq. (5) compared to $\alpha$ as measured in the field should give a rough idea of the error which deviations from simple folding will introduce into the orientation of the ancient magnetization vector.

A word of caution should be inserted here regarding the application of a plunging fold correction. Since the fold has obviously suffered a rotation about a horizontal axis, there may be reason to suspect that it has also suffered some rotation about a vertical axis as well, changing the declination of the fold axis. If known, such a rotation could be easily handled by an extension of the above method, but recovering the original attitude of the fold axis from measurements in the field may be difficult or impossible in some cases.

A FORTRAN IV subroutine which accomplishes the removal of a plunging fold and performs a simple fold test is available upon request from the author.

D. Other applications: Other applications for orthogonal transformations in paleomagnetic studies are numerous, and three brief examples will suffice to indicate their variety. Detailed derivations follow similarly to those given previously and will be omitted.

Most paleomagnetic samples are collected in such a way that the reference plane is the $x y$ plane in the sample coordinate system. In some cases, this may not be possible, and a transformation to the reference coordinate system is needed before transforming to the site coordinates.

The following procedure allows this:

1) Orient the sample such that its $z$ axis is vertical, with the oblique reference plane on top. Find and mark the new strike and $\operatorname{dip}(\beta)$ of the reference plane in this orientation.

2) Find $\Phi$, the angle between the sample $y$ axis and the new strike as measured above. Find $\Omega$, the angle between the strike as measured at the sampling site and the new strike as measured above. The angle $\Omega$ should be measured in the reference plane. See Fig. 5 for the positive sense in measuring these two angles.

3) Use:

$$
\begin{aligned}
& \phi=\Phi, \\
& \theta=-\beta, \\
& \phi=-\Omega
\end{aligned}
$$




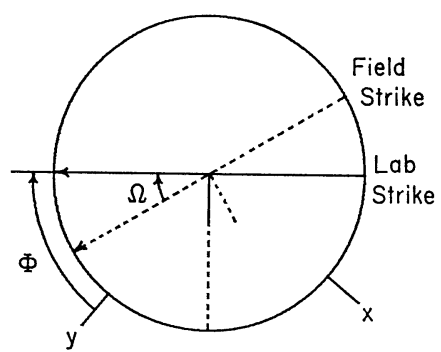

Fig. 5. Measurements needed to transform sample coordinates when a reference plane is used that is oblique to the sample $x y$ plane. The angle $\Phi$ is measured in the sample $x y$ plane, but the angle $\Omega$ is measured in the reference plane.

to transform to the reference plane coordinate system. The procedure detailed in application A may now be employed to transform to site coordinates.

Paleopole positions are often displayed in equal-area projections. It is frequently desirable to be able to center these projections about some point other than the north or south pole. This is easily accomplished by converting each latitude, longitude pair to Cartesian coordinates $(z$ axis points to north pole, $x$ axis points to Greenwich meridian) and transforming via:

$$
\begin{aligned}
& \phi=-\Phi, \\
& \theta=90^{\circ}-\Theta, \\
& \phi=180^{\circ},
\end{aligned}
$$

where $\Phi$ is the east longitude and $\Theta$ the north latitude of the point on which the plot is to be centered. The transformed coordinates are reconverted to spherical coordinates and are plotted in a Lambert equal-area projection as though it were a polar plot. Figure 6 shows a plot which has been centered on the University of Pittsburgh. To accomplish it, more than 50000 latitude-longitude pairs representing the continental outlines were transformed as described above. A PDP-10 computer required about 90 seconds to accomplish the task.

As a final example, the line connecting London to Caracas in Fig. 6 is a great circle and was generated in the following manner:

1) One of the endpoints of the line (say, London) was first transformed to the north pole via:

$$
\begin{aligned}
& \phi=\Phi_{1}, \\
& \theta=90^{\circ}-\Theta_{1}, \\
& \phi=0,
\end{aligned}
$$

where $\Phi_{1}$ and $\Theta_{1}$, are the longitude and latitude of London.

2) The great circle joining London and Caracas now lies along a meridian, so a series of points are generated with the same transformed longitude as Caracas and evenly spaced latitude from the transformed latitude of Caracas to $+90^{\circ}$ North. 


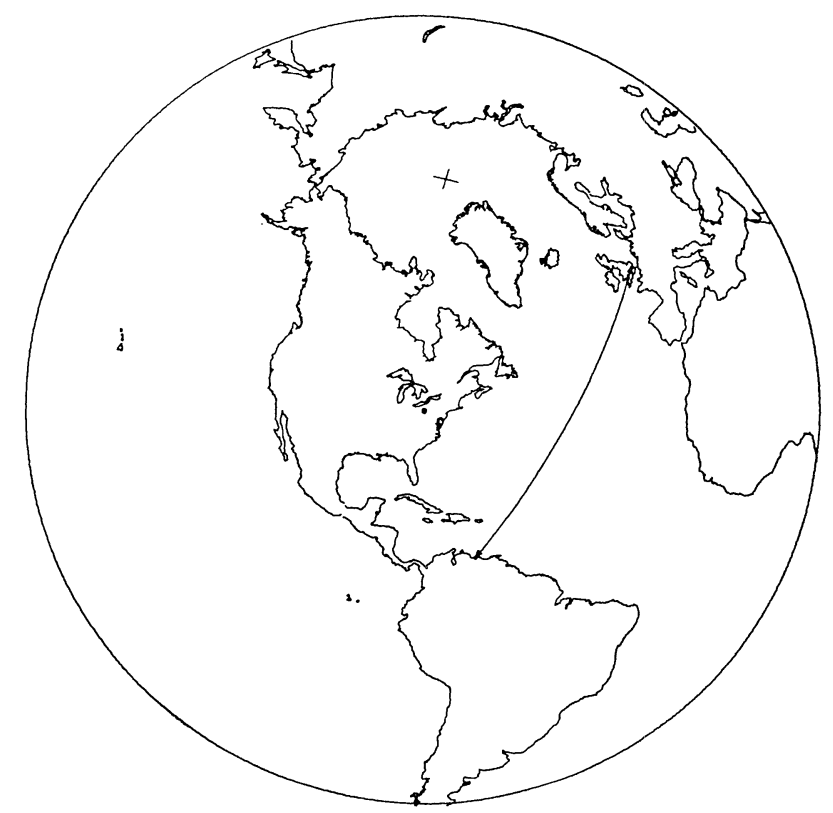

Fig. 6. A Lambert equal-area projection centered upon the University of Pittsburgh. The line connecting London and Caracas is a great circle route. The entire diagram was generated by a computer-driven $x y$ plotter using the transformations described in this paper.

3) Each of these points is now transformed back to the original coordinate system via the inverse of the first transformation:

$$
\begin{aligned}
& \phi=0, \\
& \theta=\Theta_{1}-90^{\circ}, \\
& \phi=-\Phi_{1} .
\end{aligned}
$$

Note that in inverting the transformation (11) it is necessary to reverse the order of the individual rotations. Transformation (10) is then applied to put the great circle points where they belong in Fig. 6.

\section{Summary}

The orthogonal transformation (1) is an extremely potent tool in dealing with rotations of coordinate systems. Its use is easily learned, and once its essential features are grasped, it may be used routinely to solve extremely complex problems involving rotations. Used in conjunction with a high-speed computer, the transformation can execute the most complex rotations discussed above to high accuracy in a very small fraction of a second.

The transformation thus serves essentially the same purpose as graphical 
methods based on the stereographic net, and as such has wide potential application in structural geology.

Indeed, its applications are so broad that we have barely touched upon them in this paper. As a final example, we may note that Figs. 1-4 of this paper were largely drawn by a computer-driven $x y$ plotter: the diagrams were generated as a series of lines and circles in Cartesian coordinates and then subjected to one or more transformations before plotting.

The author is grateful to Dr. Arthur Tarr of ESSA and the Naval Oceanographic Office for the use of their 110000 point geography tape, used in producing Fig. 6. This work was supported under grants from the National Science Foundation.

\section{REFERENCES}

Goldstein, H., Classical Mechanics, Addison-Wesley, Reading, Massachusetts, U.S.A., 1950. ZotKevich, I.A., Transformation of the coordinates of the vector of the natural remanent magnetization in paleomagnetic studies, Izv. Earth Physics, No. 6, 104, 1966.

Zotкevich, I.A., Reduction of the natural remanent magnetization of a plunging fold to the ancient coordinate system in paleomagnetic studies, Izv. Earth Physics, No. 2, 95, 1972. 